\title{
Characteristics of effective interventions supporting quality pain management in Australian emergency departments: An exploratory study
}

\author{
Ramon Z. Shaban, RN PhD FRCNA ${ }^{a, b, d, *}$, \\ Kerri Holzhauser, RN BN ${ }^{a, c}$, \\ Kerri Gillespie, BA BPsych PGDipPsych ${ }^{d}$, \\ Sue Huckson, B AppSci, RN ICU Cert ${ }^{e}$, \\ Scott Bennetts, RN BHSc (Nursing) GDipCritCareNurs GCertHealthProm ${ }^{\mathrm{e}}$
}

\footnotetext{
a Griffith Health Institute, Research Centre for Clinical and Community Practice Innovation, Griffith University, Australia

b Department of Emergency Medicine, Princess Alexandra Hospital, University Drive, Meadowbrook, QLD 4131, Australia

c Nursing Practice Development Unit, Princess Alexandra Hospital, Australia

' School of Nursing and Midwifery, Griffith University, University Drive, Meadowbrook, QLD 4131, Australia

e National Health and Medical Research Council National Institute of Clinical Studies, Level 1, Fawkner Centre, 499 St Kilda Road, Melbourne, Vic 3004, Australia
}

Received 22 September 2011; received in revised form 30 November 2011; accepted 30 November 2011

\author{
KEYWORDS \\ Pain; \\ Management; \\ Emergency \\ departments; \\ Evidence; \\ Quality improvement
}

\begin{abstract}
Summary
Background: It is well established that pain is the most common presenting complaint in Emergency Departments. Despite great improvements in available pain management strategies, patients are left waiting for longer than $60 \mathrm{~min}$ for pain relief on arrival to the emergency department. The aim of this study was to describe interventions that lead to successful implementation of the National Health and Medical Research Council approved guidelines Acute Pain Management: Scientific Evidence (2nd Edition) that include specific recommendations for best practice pain management.

Methods: A two-phased, mixed-method, exploratory study of all 52 Australian hospital emergency departments participating in the National Emergency Care Pain Management Initiative incorporating interview and document analysis was undertaken.
\end{abstract}

\footnotetext{
* Corresponding author at: Griffith Health Institute, Research Centre for Clinical and Community Practice Innovation, University Drive, Meadowbrook QLD 4131, Australia. Tel.: +61 73382 1271; fax: +61 733821277.

E-mail address: r.shaban@griffith.edu.au (R.Z. Shaban).
}

1574-6267/\$ - see front matter @ 2011 College of Emergency Nursing Australasia Ltd. Published by Elsevier Ltd. All rights reserved. doi:10.1016/j.aenj.2011.11.003 
Findings: Interventions used by clinicians to improve pain management included nurse initiated analgesia, intranasal fentanyl for paediatric patients and lignocaine, and facio illiaca block. Education formed a major part of the intervention and the development of a working group of key stakeholders was critical in the successful implementation of change. Staff perceptions of patients' pain level and attitudes toward pain assessment and pain management were identified as barriers.

Conclusion: This study highlighted how an effective framework to plan and implement practice change and tailored interventions, including education and training systems and products using the best available evidence, best equipped clinicians to manage pain in the ED.

(c) 2011 College of Emergency Nursing Australasia Ltd. Published by Elsevier Ltd. All rights reserved.

\section{What is known}

- Pain is the most common presenting patient complaint in Emergency Departments (ED) globally, making pain relief an imperative goal.

- Despite great improvements in analgesia medication, numerous studies report that pain management by ED staff is still insufficient and inconsistent.

- Little is known about the specific interventions that lead to successful implementation of the evidence for pain management in emergency care settings.

\section{What this paper adds}

- Enablers to the uptake of evidence included targeted education, change champions and leaders, and new clinical guidelines and policies specific to practice change.

- Barriers included workloads, demand for service delivery, space, and equipment, organisational governance, the lack of interdisciplinary collaboration, and developing complicated clinical guidelines.

\section{Introduction}

It is well established that pain is the most common presenting patient complaint in Emergency Departments (ED) globally, ${ }^{1-5}$ making pain relief an imperative goal. Despite great improvements in analgesia medication, numerous studies report that pain management by ED staff is still insufficient and inconsistent. ${ }^{1,6-8}$ Patients with moderate to severe pain are often left to wait for longer than 60 min on average ${ }^{5,9}$ with some studies reporting mean times between $74^{3}$ and $131^{10}$ min for analgesia, and up to a third remain unrelieved on discharge. ${ }^{6}$ Pain scores are poorly documented and pain is rarely re-assessed. ${ }^{9}$ Children receive fewer analgesics than adults, ${ }^{6,11}$ and a number of other factors, including gender, ethnicity, and age have been suggested as effecting time to, and access to analgesia. ${ }^{2}$ The National Health and Medical Research Council of Australia have estimated that the cost of unrelieved pain in 2009 was around $\$ 514$ billion due to delayed recovery and longer inpatient stays. ${ }^{12}$
A number of reasons have been suggested as the cause for such an international deficit in pain management. Studies have clearly shown an inconsistency between nurses' beliefs about patient pain, and patients' subjective pain scores. ${ }^{6}$ Nurses have been seen to frequently underestimate patient pain, especially when an obvious physical abnormality cannot be found. ${ }^{7,13}$ A study in $2008^{8}$ revealed a discrepancy of almost $50 \%$, with the majority of these nurses underestimating patient pain by one category (on a $0-10$ numerical rating scale). Poor education of pain management was also seen to be a common situation among ED nurses. ${ }^{1}$ Many ED staff and patients cultivate the fear that opioid provision will lead to dependence, ${ }^{4,14}$ and medical staff often show reluctance to distribute these medications on suspicion of drug seeking behaviour by patients. ${ }^{6}$ Crowding in the ED is another barrier to pain management, with an increased number of patients lengthening the average time to analgesia. ${ }^{10,15}$

Although pain management has improved little over the past 20 years, ${ }^{14}$ there is evidence to show that there are ways to improve the current trends in pain management. Education programs have been shown to increase awareness of pain and patient anxiety. ${ }^{1}$ This increased awareness has resulted in an improvement in nurse's knowledge of analgesic options as well as pain management, and department audits and feedback have led to an increase in analgesia distribution as well as a decrease in time to analgesia in a number of studies. ${ }^{11}$

In 2007 a national audit ${ }^{16}$ was undertaken to establish current practice against the National Health and Medical Research Council approved guidelines developed by the Australian and New Zealand College of Anaesthetists. ${ }^{17}$ Significant need for practice improvement was identified. In June 2008, the National Health and Medical Research Council-National Institute for Clinical Studies Emergency Care Community of Practice launched the National Emergency Care Pain Management Initiative ${ }^{18}$ to implement the National Health and Medical Research Council guidelines for pain management improvement in Australian emergency departments. Core components of the National Institute for Clinical Studies intervention included training of local project leads in guideline implementation with provision of a specific training program, development of an organisational policy supporting pain management in the ED as a clinical priority, audit and feedback of pain management performance, development of locally relevant interventions, project lead support to provide practical support for 
project management and guideline implementation expertise, and development of a web-based data reporting system for the collation of local de-identified data and as a repository for participating teams to share resources.

\section{Aims}

The aim of this study was to describe interventions that lead to successful implementation of the National Health and Medical Research Council approved guidelines Acute Pain Management: Scientific Evidence (2nd Edition) ${ }^{17}$ that include specific recommendations for best practice pain management. The specific questions for this study were:

1. What are the characteristics of the interventions used to implement the National Health and Medical Research Council approved guidelines Acute Pain management: Scientific Evidence (2nd Edition) ${ }^{17}$ ?

2. What interventions enhanced the enablers of, and ameliorated the barriers to, the uptake of Acute Pain management: Scientific Evidence (2nd Edition) ${ }^{17}$ to improve in pain management in the emergency department?

3. What organisational characteristics act as enablers of and barriers to the uptake of the Acute Pain management: Scientific Evidence (2nd Edition) ${ }^{17}$ to improve pain management in the emergency department?

4. What level of sustainability have the interventions afforded the ongoing use of the guidelines for pain management best practice?

\section{Methods}

\section{Study design}

A two-phased, mixed-method, exploratory study of Australian hospital emergency departments participating in the National Emergency Care Pain Management Initiative incorporating interview and document analysis was undertaken.

\section{Recruitment}

Convenience sampling was taken from the 52 Australian hospital emergency departments participating in the National Emergency Care Pain Management. Project leads and key stakeholders for the National Emergency Care Pain Management Initiative from within each of the emergency departments were invited to participate. Recruitment occurred by and telephone. Of the 52 invitees, 45 EDs participated in the NHMRC NICS National Emergency Care Pain Management Initiative. Of these 45, 11 nurses, one doctor, one pharmacist and one quality manager from the nine EDs participated in this study.

\section{Data collection}

Data collection occurred September 2009-December 2010 and consisted of two phases:
Phase 1 - interviews and focus groups

Interviews were conducted with 11 nurses. The role of these participants included registered nurses, nurse practitioners, and nurse managers, of which nine were female and two were male. Semi-structured interviews with key stakeholders were undertaken to determine the utility of the invention in developing evidence implementation capacity and the quality of program delivery. Topics explored during the interview included the types initiatives implemented, barriers, and challenges to implementation, enabling aspects, hospital characteristics, outcomes, future plans, and sustainability. All interviews were transcribed verbatim and anonymised with respect to the participating individual. The transcripts were coded to enable the matching of transcripts with data obtained in phase 2 , which enable a comparative analysis. Participants were provided an opportunity to review the transcription of their interview and make adjustments and corrections, and approve the data provided.

Phase 2 - document and policy analysis

Policies, guidelines, and documents outlining infrastructure, strategies, and outcomes pertinent to the National Institute for Clinical Studies Emergency Care Pain Management Initiative were collected from participating hospitals. A list of the documents collected and analysed is included below in Table 1. All documents were anonymised and coded by staff at the National Institute for Clinical Studies (SB and $\mathrm{SH}$ ) to enable matching with interviews obtained in phase 1 by facility. Four workshops for each wave of the study were conducted to discuss current issues and evaluate progress. Workshop summary reports were collected from the National Health and Medical Research Council National Institute for Clinical Studies National Emergency Care Pain Management Initiative Website, along with communications and other documentation relevant to the study.

\section{Data analysis}

Authors RS, KH, and KG, who were blinded to the identity of the participants originating facility, conducted the analysis of data from phases 1 and 2 and the documents provided in phase 2 . The transcripts of interview and documents and policies together were analysed using content analysis according to Silverman. ${ }^{19}$

\section{Research ethics}

Approval to conduct this research was obtained from the Griffith University Human Research Ethics Committee.

\section{Findings}

\section{Interventions were by participants and facilities}

The participants reported that the National Emergency Care Pain Management Initiative resulted in the uptake of various implementation strategies. The most common intervention was the introduction of nurse-initiated analgesia. Other interventions included the introduction of new drugs, 
Table 1 Documents used.

Hospital documents:

- Austin Health Emergency Dept (2009) - Guidelines for Nurse-initiated pain management in adults

- Austin Health (July 2009) - Nurse initiated analgesia for adults E-learning module (includes background on PM, Nurse Initiated Analgesia standing order, assessing pain, Pharmacology, patient scenarios, and competency assessment)

- Canberra Hospital - Summary of policy, infrastructure and people (e.g. formalised pain scales, Laceraine administration, introduction of drug cupboard at triage, etc.)

- Joondalup Health Service - Triage adult and paediatric pain pathways

- Mackay Health Service District (Nov 2008) - Guidelines for Nebulised Lignocaine for Nasogastric Tube Insertion

- Port Macquarie Base Hospital (2009) - Guidelines for Methoxyflurane (PENTHROX) administration

- Princess Margaret Hospital for Children, Perth (October 2006) - Guidelines for Intranasal Fentanyl administration

- Princess Alexandra Hospital - Guidelines for Nurse Initiated Analgesia

- Princess Alexandra Hospital - Acute pain guidelines

- Princess Alexandra Hospital - Procedure Manual for Nurse Initiated Analgesia and Nurse Initiated Medications

- Sir Charles Gardner Hospital - Nurse Led Analgesia protocol and guidelines

- Southern Health (September 2009) - Resource Package for Standing Order - Nurse Administered Analgesia

- The Townsville Hospital (July 2008) - Guidelines for Nebulised Fentanyl for pain management in young children

- Western Health - Policy and procedure for administration of an IV opioid bolus.

- Western Health - Pain management package (includespain assessment scales, opioid administration Pethidine Hydrochloride administration, and assessment tool)

- Western Health - Guidelines for Nurse Initiated Analgesia

- Werribee Mercy Hospital (Sept 2009) - Guidelines for Intranasal Fentanyl (Paediatric and Adult) administration

- Werribee Mercy Hospital (2007) - Assessment (for completion before accreditation in the use of nurse initiated pain management)

- Werribee Mercy Hospital - Guidelines for Nurse Initiated Medications

Other documents:

- Baseline data analysis - Scott Bennetts' analysis of wave one and wave two baseline findings

- Wave one data analysis (December, 2009)

- NHMRC - Barriers and enablers to pain management in emergency department care: a literature review (July, 2008)

- NHMRC/NICS - Audit of Pain Management Practices in Emergency Departments across Australia (August, 2007)

- NICS - Qualitative Research: Implementation of best practice pain management in emergency departments (December, 2007)

- NHMRC/NICS pain management workshop overviews (wave one and two)

- Website https://emergencycare.nhmrc.gov.au/pain/index.php - much of the above data was gained from the website

such as methoxyflurane, intranasal fentanyl, lignocaine, and facio illiaca block. Education for staff about these was common to all participating facilities. This was reported to be critical for practice change, as it increased clinicians awareness, knowledge of pain, and pain management practices within the ED, as exemplified in the following excerpt of talk:

On a personal level I've really enjoyed it. It's challenged my personal views of pain relief ... And just looking at patients' pain in a new light again; I found that really refreshing and really good for me professionally.

Training packages for attending to patients in pain were produced in many of the departments. These outlined the information required for the administration of analgesia and highlighted those staff members who were approved to administer pain medication. The administration of analgesia to patients was dependent on pain scores, further emphasizing the importance of pain score assessment in the ED. Instructions were clear and detailed with specific pain medications being prescribed for different pain scores. Although none of the resources recommended the consideration of different injury types when choosing which analgesia to use, the instructions did clearly indicate contraindications and exclusionary criteria. Only a very small number of these resource packages included any detailed information outlining background information on pain management in the $E D$, or the evidence base for implementing such guidelines. A key feature of the documents and their use as reported by the participants was the inclusion of detailed pain score instructions, which directing nurses to the correct protocols for addressing pain. Consistent guidelines on pain scores were implemented, pain scoring charts were made more available and visible to staff, and in some departments were made compulsory at triage. All were accompanied by detailed descriptions of approved administrators of these analgesics, as well as instructions for this administration. Analgesia used, and protocols differed slightly across hospitals, but the objectives and final actions recommended were similar. These documents indicated that the timely and accurate assessment of pain followed by an appropriate and effective administration of analgesia while observing all safety measures were best practice.

\section{Enablers and barriers to the uptake of the evidence}

Several themes emerged which represent the major issues for ED employees when implementing improvements to pain 
management within their department. The first, and most important, was the perception of staff.

\section{Staff perceptions about existing practices and the need for change}

Participants cited staff perceptions as both a guiding force for change and a barrier. Staff's perceptions affected how much they accepted change, and how they conducted pain management. In order to improve pain relief procedures, it was understood that staff must recognise deficiencies in existing pain management practices:

\section{"Before we started with the project we thought we man- aged pain very well, and we thought we did it in a timely fashion and we thought we were using the best appro- priate methods.",}

Participants reported feeling that their hospitals were doing a good job. These perceptions were thought to be a barrier to previous change as staff did not consider change necessary until audits were conducted. Most participants were surprised to see the results of the first audit, and were disappointed at the poor results.

"Everyone thought they were doing a good job. And they were very, very surprised to see that they were actually doing so badly.",

One way of altering staff perceptions was to provide feedback. This included the feedback from audit reports, showing clearly the length of time to analgesia currently seen in the ED, and the poor levels of documentation and pain relief for patients before discharge. This feedback was important as a motivator. However, feedback on improvements at the second and third data collection points proved to be encouraging for staff, and helped sustain the change. Participants considered the results of the audits to be useful. One participant reported that it was a 'non-confrontational' way of informing staff of the limitations of their practice. One participant reported that that once initiatives were put in place, the results themselves would help to alter staff perceptions, and therefore staff behaviour.

To be able to provide pain relief to a child without having to sedate them and have their length of stay reduced is a win for both patients and staff in the department ... When they can see the results, for the INF for instance, instead of having to hold a kid down or inflict pain, you give them the intranasal fentanyl, and they just sit there, they're like putty in your hand... It's less traumatic for the child, it's less traumatic for the parent, and at the end of the day it makes the whole process easier and the patient benefits.

Staff perceptions included a number of beliefs that were unfounded, but affected delivery of pain relief. Education was reported to be a good way of creating greater awareness of what they could and could not do within the confines of pain management.

The old surgical myth of don't give pain relief to a surgical patient awaiting surgical review in case you hide the symptoms. . . and also the myth about worrying about whether or not patients will become dependent on opioids.

Another interconnected theme was the attitudes staff had towards pain relief, in particular to the indications for pain relief and the way in which pain was managed.

\section{Staff attitudes towards practice improvement}

Participants reported that nurses were not willing to accept patient's subjective pain ratings, especially when the source of the pain was not obvious or when the patient seemed 'visually' to be in less pain.

I've seen an old lady come in with her arm in a sling saying that she had 10 out of 10 pain, and I must admit I didn't believe her ... and when I saw the X-ray ... she had a dislocated shoulder. So she did have [pain] but she didn't present to my eyes like someone who was in 10 out of 10 pain.

I think that it did highlight to other staff members ... how subjective pain is, and how important it is to believe the patient and go with that.

Related to the subjectivity of pain assessment was the reported 'burn out' or the desensitisation of staff towards patients in pain due to pressures of workload. The experienced staff were not as enthusiastic about implementing practice change and were less willing to change their current practices, as illustrated in the following excerpts of talk:

One of our main barriers has been burnt out staff with lack of empathy for patients, and I think that's universal among both medical and nursing staff, who've seen a lot of it and are working under big pressures, and pain management is just one too many things for them and they don't have enough empathy to see the pain and address it quickly.

I think the biggest barrier for any pain initiative is changing people's hearts... It's about making people care and have empathy ... the older the clinician the less likely they are to care about patients.

\section{Organisational characteristics acting as enablers and barriers}

The theme 'organisational structure' defined aspects of the department which affected the ease or difficulty with which changes could be made or upheld.

\section{Oganisational structure within the ED}

Having co-operation, both within the nursing team, and between departments was important for the successful implementation of pain management initiatives. Participants who had the full co-operation of key members of the hospital, executive staff, of pharmacy and of medical records found all aspects of the study, as well as the implementation of their initiatives was much easier. On the other hand, some participants reported that the clinical governance of the hospital and lack of engagement across 
divisions were barriers to implementing and maintaining change. One participant reported that that process within the hospital required for implementing any form of change was so slow and demanding, that it deterred any attempt to improve current services or procedures.

\section{Resources - time and staffing}

Time was reported to be the most significant barrier to pain management. This included time to assess patients, time to complete chart audits, and time to get analgesia for patients, as the following excerpt of talk data illustrates:

Don't have time to get pain scores or analgesia.

Staffing was significant barrier to the uptake of evidence. A high staff turnover rate and staff working long hours and shift work were reported to affect pain management practices. Another two participants reported unco-operative and uncommitted team members. It was thought that in some instances this may be caused by the excess workload of the data collection.

'It's taken people's enthusiasm and time away from actually introducing initiatives.",

\section{Maintenance and sustainability of the interventions}

When asked about the sustainability of their project, most people reported the use of regular auditing and education. The majority of participants had introduced pain management training into nursing staff orientation and education modules, as well as implementing monthly case histories. Most participants reported that the introduction of education, due to the faster acceptance of new staff members, would mean sustainability in the long term for improved pain management. Education was reported in the workshops as an important factor for sustainability. A number of resource packages were created for staff, many of these including self assessments.

\section{Discussion}

The findings of this study have implications for (i) change management and practice improvement processes in clinical practice; (ii) education and training; (iii) policies and procedures; and (iv) organisational governance.

\section{Practice change}

Planning is critical for effective practice change. ${ }^{20}$ Using an effective framework to plan and implement practice change has been identified as critical by the participants in this study. The approach for practice change by the participants consisted of identification of the evidence based practice change, developing locally relevant interventions based on the evidence, analysis of barriers and enablers for change within their organisation and the development of strategies to manage these barriers and enablers. ${ }^{18}$ The barriers identified from this study support the existing literature regarding the deficit in pain management. The first includes staff subjective attitudes toward patients reported pain, ${ }^{6}$ although there was no mention from participants relating to the underestimation of pain, 7,13 there was some perception by participants that certain injuries do correlate with expected severity of pain. For example, a patient with a dislocated shoulder should have severe pain. Other staff perceptions related to pain management include the belief of the provision of opioids leads to dependence, ${ }^{4,14}$ and this has been supported by the results. One perception that has been identified is the myth that pain management should not be instigated until surgical review. There is now supporting evidence to support the initiation of pain management for patients awaiting review. ${ }^{21}$

Using a framework to identify barriers and enablers within the clinical area assisted the staff to develop strategies to facilitate a more effective practice change. Some of these strategies included providing feedback regarding current practice and results as practice change was implemented, developing teams that included staff that were keen to participate, developing relevant organisational supporting documentation around the practice change, and communication strategies to feedback to staff.

For a successful practice change, change management theory identifies that strategies for sustainable change are essential. ${ }^{20}$ Sustainability of the practice change beyond the life of the project was a high on the participant's agenda. To support the sustainability, practice change included changes to documentation and electronic management of patient data. Data collection documentation was updated to include the relevant changes, for example including a place for compulsory recording of a pain score.

\section{Education and training}

The literature indicates that ED nurses report the provision of pain management education as poor. ${ }^{1}$ There is a popularly held fear amongst ED staff, policy makers and patients fear that provision of analgesia, in particular opioids, will lead to dependence. ${ }^{4,14}$ Moreover, staff are often reluctant to administer such medications out of suspicion of drug seeking behaviour by particular patients. ${ }^{6}$ This study highlights the importance of tailored education and training systems and products to equip clinicians for managing pain in the ED in accordance with the best available evidence. In the patient context, the success of education for have been shown to increase awareness of pain and patient anxiety. ${ }^{1}$ The success of interventions for pain management initiatives, such as nurse-initiated analgesia, is contingent on education systems and products that provide clinician with robust, up-to-date evidence.

\section{Organisational governance}

It is well known that organisational factors affect change management processes. These include organisational structure, culture and economic factors. ${ }^{20}$ The participants identified that a variety of issues including organisational culture, the size of the organisation occurred within the organisational governance that had the potential to impact on the effectiveness of the practice change. Developing teams that included as many relevant disciplines both within the unit and across the organisation were more successful 
in the practice change and its sustainability than those that did not.

There are important limitations to note when considering the findings of this study. First, the study is based on a convenience sample of nine EDs in Australia, and the findings are not generalisable to all EDs. Second, the convenience sample compromise EDs who were actively engages in practice improvement with respect to pain management. This study does not account for other EDs who are not similarly engaged, or those with other priorities. Third, the data provided during interview was self-reported, and it cannot be assumed that this reflects actual clinical practice.

\section{Conclusions}

This study highlighted how an effective framework to plan and implement practice change and tailored interventions, including education and training systems and products using the best available evidence, best equipped clinicians to manage pain in the ED. Enablers to the uptake of evidence included targeted education, change champions and leaders, and new clinical guidelines and policies specific to practice change, and barriers included workloads, demand for service delivery, space, and equipment, organisational governance, the lack of interdisciplinary collaboration, and developing complicated clinical guidelines. Enabling multidisciplinary teams within individuals units and the wider organisation lead and effect sustainable practice change favoured improved outcomes.

\section{Provenance and Conflict of Interest}

Ramon Z. Shaban is Editor-in-Chief of the Australasian Emergency Nursing Journal but had no role in the peer review and editorial decision-making of this manuscript whatsoever. Dr Shaban is a member of the National Health and Medical Research Council National Institute of Clinical Studies Emergency Care Community of Practice Pain Management Initiative Advisory Committee. He has no other competing interests or conflict of interests to declare.

Kerri Gillespie has no competing interests or conflict of interests to declare.

Kerri Holzhauser is an Associate Editor of the Australasian Emergency Nursing Journal but had no role in the editorial review of this manuscript whatsoever. Mrs Holzhauser is a member of the National Health and Medical Research Council National Institute of Clinical Studies Emergency Care Community of Practice Pain Management Initiative Advisory Committee. She has no other competing interests or conflict of interests to declare.

Sue Huckson is Director of the Effective Practice Program at the National Health and Medical Research Council National Institute of Clinical Studies. She is a member of the National Health and Medical Research Council National Institute of Clinical Studies Emergency Care Community of Practice Pain Management Initiative Advisory Committee. She has no other competing interests or conflict of interests to declare.

Scott Bennetts is Assistant Director of the Effective Practice Program at the National Health and Medical Research Council National Institute of Clinical Studies. He is a member of the National Health and Medical Research Council National Institute of Clinical Studies Emergency Care Community of Practice Pain Management Initiative Advisory Committee. He has no other competing interests or conflict of interests to declare. This paper was not commissioned.

\section{Funding}

This paper was supported by funding awarded by the National Health and Medical Research Council National Institute for Clinical Studies.

\section{Human research ethics approvals}

Approved to conduct this research was granted by the Griffith University Human Research Ethics Committee. The research was conducted in accordance with the approved protocol.

\section{References}

1. Augarten A, Zaslansky R, Pharm IM, Minuskin T, Lerner-Geva L, Hirsh-Yechezkel G, et al. The impact of educational intervention programs on pain management in a pediatric emergency department. Biomedicine and Pharmocotherapy 2006;60(7):299-302.

2. Johnson SH. Introduction pain management in the emergency department: current landscape and agenda for research. The Journal of Law, Medicine and Ethics 2005;33(4):739-40.

3. Safdar B, Heins A, Homel P, Miner J, Neighbor M, DeSandre P, et al. Impact of physician and patient gender on pain management in the emergency department - a multicenter study. Pain Medicine 2000;10(2):364-72.

4. Thomas T. Providing pain relief for patients in the emergency department. Art and Science Emergency Care 2007;22(9):41-5.

5. Todd KH, Ducharme J, Choiniere M, Crandall CS, Fosnocht DE, Homel $P$, et al. Pain in the emergency department: results of the pain and emergency medicine initiative (PEMI) multicenter study. The Journal of Pain 2007;8(6):460-6.

6. Bauman BH, McManus JG. Pediatric pain management in the emergency department. Emergency Medicine Clinics of North America 2005;23(2):393-414.

7. Ducharme J. Clinical guidelines and policies: can they improve emergency department pain management? Journal of Law, Medicine, and Ethics 2005; (Winter):783-90.

8. Duignan M, Dunn V. Congruence of pain assessment between nurses and emergency department patients: a replication. International Emergency Nursing 2008;16(1):23-8.

9. Holdgate A, Shepherd SA, Huckson S. Patterns of analgesia for fractured neck of femur in Australian emergency departments. Emergency Medicine Australasia 2010;22(1):3-8.

10. Forero R, Mohsin M, McCarthy S, Young L, leraci S, Hillman K, et al. Prevalence of morphine use and time to initial analgesia in an Australian emergency department. Emergency Medicine Australasia 2008;20:136-43.

11. Herd DW, Babl FE, Gilhotra Y, Huckson S. Pain management practices in paediatric emergency departments in Australia and New Zealand: a clinical and organizational audit by National Health and Medical Research Council's National Institute of Clinical Studies and Paediatric Research in Emergency Departments International Collaborative. Emergency Medicine Australasia 2009;21(3):210-21.

12. Duignan M, Dunn V. Perceived barriers to pain management. Emergency Nurse 2009;16(9):31-5. 
13. Wilsey BL, Fishman SM, Ogden C, Tsodikov A, Bertakis KD. Chronic pain management in the emergency department: a survey of attitudes and beliefs. American Academy of Pain Medicine 2008;9(8):1073-80.

14. Hogan SL. Patient satisfaction with pain management in the emergency department. Topics in Emergency Medicine 2005;27(4):284-94.

15. Hwang U, Richardson LD, Sonuyi TO, Morrison RS. The effect of emergency department crowding on the management of pain in older adults with hip fracture. Journal of the American Geriatrics Society 2006;54(2):270-5.

16. Yeoh M, Huckson S. NICS EC COP Audit of Pain Management ACEM report 2007; 2007, http://www.nhmrc.gov.au/nics/ nics-programs/national-emergency-care-pain-managementinitiative [accessed 1st April 2010].

17. Australian and New Zealand College of Anaesthetists, Faculty of Pain Medicine. Acute Pain Management: Scientific Evidence, 2nd Ed. Melbourne: Australian and New Zealand College of Anaesthetists, 2005.

18. National Health and Medical Research Council. National Institute for Clinical Studies emergency pain management initiative; 2008, http://www.nhmrc.gov.au/nics/nics-programs/ national-emergency-care-pain-management-initiative [accessed 1st April 2010].

19. Silverman D. Interpreting qualitative data: methods for analysing talk, text and interaction. 3rd ed. London: SAGE; 2008.

20. Robbins SP, Millett B, Waters-Marsh T. Organisational behaviour. Frenchs Forest Pearson Prentice Hall; 2004.

21. Macintyre PE, Schug SA, Scott DA, Visser EJ, Walker SM, APM:SE Working Group of the Australian and New Zealand College of Anaesthetists and Faculty of Pain Medicine. Acute Pain Management: Scientific Evidence. 3rd edition Melbourne: ANZCA \& FPM; 2010. 